\title{
Von der Rationalität des Rechts in die Irrationalität der Sicherheit
}

- Reflexionen über Widerstandsformen in Sicherheitsgesellschaften -

Dokumentation einer Podiumsdiskussion anlässlich der Leipziger Buchmesse im Historischen Plenarsaal des Bundesverwaltungsgerichts am 18. März 2010 - Moderation Michael Voß -

A. Rechtserosionen und Widerstandsformen - Eine Zuführung (Michael Voß)

B. Die Entwicklung der Erosionen zentraler Rechtsprinzipien (Peter-Alexis Albrecht)

C. Zur Geschichte paradigmatischer Widerstandsformen

I. Georg Elser: Widerstand in der NS-Zeit (Ulrich Renz)

II. Widerstandsformen in der DDR (Hans Misselwitz)

III. Widerstandsformen in der BRD (Karl-Heinz Dellwo)

D. Zur Erforderlichkeit und Angemessenheit legitimer Widerstandsformen gegen die Sicherheitsgesellschaft (Michael Voß)

\section{A. Rechtserosionen und Widerstandsformen - Eine Zuführung (Michael Voß)}

Die Podiumsdiskussion steht unter dem Titel „Der Weg in die Sicherheitsgesellschaft“. Wir wollen die gleichnamige Neuerscheinung von Peter-Alexis Albrecht aus dem Berliner Wissenschaftsverlag ${ }^{1}$ zum Anlass nehmen, über einen drohenden Verfall des Rechtsstaats auf der einen Seite und die Frage, wie einer solchen Entwicklung widerstanden werden kann auf der anderen Seite, zu diskutieren.

Nach dem Vortrag der Ausgangsthese Albrechts, dass wir uns auf dem Weg in eine Sicherheitsgesellschaft befinden, in der die Beseitigung rechtsstaatlicher Grundprinzipien droht, werden die übrigen Beteiligten gebeten, in Bezug auf die von ihnen erlebten bzw. recherchierten historischen Widerstandserfahrungen - auch im Hinblick auf die Gewaltfrage - Schlussfolgerungen für die vorab skizzierten Gesellschaftsgefahren zu ziehen. Wir wissen, dass die Erfahrungsbereiche der hier auf dem Podium versammelten Personen höchst heterogen sind. Wir wollen mit diesen Widerstands-Beispielen auch nicht auf eine historische Kontinuität oder historische Reihenfolge verweisen. Es sind höchst unterschiedliche, zum Teil dramatische Erfahrungsbereiche mit Widerstand gegen den Staat oder staatliche Institutionen. Bisweilen führen aber gerade dramatische Ereignisse in der Rückschau zu Erkenntnissen, die im politischen Alltag verschüttet sind. An diese Erkenntnisse wollen wir heran.

1 „Der Weg in die Sicherheitsgesellschaft - Auf der Suche nach staatskritischen Absolutheitsregeln" (Berliner Wissenschaftsverlag 2010). 
Um welche Widerstandsbereiche wird es gehen:

Ulrich Renz ${ }^{2}$ als Elser-Biograph verweist darauf, dass Georg Elsers Bomben-Attentat gegen die Spitzen des NS-Staates (8. November 1939) kein Vorbild für Zeiten ist, in denen es andere Widerstandsmöglichkeiten gibt. Es verkörpert aber das Widerstandsrecht, das jedermann zusteht. Renz insistiert auf der Subsidiarität des Widerstandsrechts gegenüber anderen rechtlichen oder parlamentarischen Wegen. In der Bewertung des Elser-Attentats wird deutlich, dass die Klassifizierung von „Widerstand“ mit der „Definitionshoheit" zu tun hat, die meist in den Händen des Staates liegt, gegen den sich der Widerstand richtet. Beim aufgeklärten Publikum überwiegt heute die Deutung, wonach hier ein mutiger, berechtigter, dem Täter zur Ehre gereichender Versuch vorliegt, durch ein Attentat auf Hitler das Schlimmste zu verhindern. Aber noch in der jüngsten politologischen Diskussion changiert das Elser-Attentat zwischen „notwendigem Befreiungsschlag“ und „moralisch nicht gerechtfertigtem Gewaltakt".

Hans Misselwitz ${ }^{3}$ geht davon aus, dass in einem totalitären Regime alles und jedes Verhalten als Widerstand eingestuft und unterdrückt werden kann. Widerstand hat also viel mit Zuschreibung und der Definitionshoheit des Staates zu tun. Insofern kann „Widerstand" von einem Notwehrinstrument des Bürgers zu einem Eingriffsinstrument des Staates werden. Überträgt man das auf unser Thema, dann könnte etwa die berechtigte Anrufung des Verfassungsgerichts gegen staatliche Sicherheitsgesetze auch als unzulässiger „Widerstand“ (gegen die notwendige Terrorismusbekämpfung) eingestuft und auf diese Weise desavouiert werden.

Karl-Heinz Dellwo ${ }^{4}$ stellt auf die strukturellen Ursachen von Problemlagen ab, gegen die Widerstand geradezu zwingend gefordert wird. Er sieht die Chancen für die Mobilisierung von Widerstand eher bei den von sozialen Problemen direkt Betroffenen. Dellwo kritisiert die Selbstisolierung von (gewaltsamen) Widerstandsgruppen, die Selbstbezüglichkeit der Akteure, die nur mit sich selbst beschäftigt sind und den gesellschaftlichen Gegenstand ihrer Widerstandsbewegung aus dem Auge verlieren.

Wir können und wollen diese höchst unterschiedlichen Erfahrungsbereiche nicht in ihrer historischen Komplexität entfalten. Jeder Bereich für sich hat ganze Konferenzen mit Themen versorgt und viele Regalmeter wissenschaftlicher Literatur zur Folge gehabt. Im Folgenden kann es nur um wenige übergreifende Aspekte des Widerstandsthemas gehen, beispielsweise um Fragen

- zur Erforderlichkeit: Ist ein wie immer gearteter politischer Widerstand gegen eine gesetzgeberische Erosion des Rechtsstaats erforderlich - über die bereits eingeschlagenen Rechtswege hinaus? Genügt es nicht, auf die gerichtliche Überwachung der rechtsstaatlichen Grundprinzipien zu vertrauen?

2 Ulrich Renz, Journalist und Autor, langjährig Chefredakteur von Associated Press, hat über zahlreiche NS-Gerichtsprozesse berichtet, war Gerichtsberichterstatter beim Bundesverfassungsgericht. Renz hat in vielen Beiträgen über den Hitler-Attentäter Georg Elser publiziert.

3 Hans Misselwitz, Mitglied der Friedensbewegung in der DDR, stellvertretender Außenminister in der - etwas kurzlebigen - de Maizière-Regierung (1990), auch Buchautor über seine Erfahrung in und mit der Wende.

4 Karl-Heinz Dellwo war an der Geiselnahme in der Deutschen Botschaft in Stockholm 1975 beteiligt, bei der zwei Botschaftsangehörige erschossen wurden. Dellwo war von 1975 bis 1995 inhaftiert. Er arbeitet heute als Autor und Dokumentarfilmer in Hamburg. 
- zur Angemessenheit der Mittel: Was sind hier und heute angemessene und legitime Widerstandsformen gegen eine Rechtsentwicklung, die den Rechtsstaat im Kern gefährdet?

- zur Anwendung von Gewalt: Welche Schlussfolgerungen können aus den historischen Beispielen zur Frage der Berechtigung - oder Verwerfung - eines gewaltsamen Widerstands gezogen werden?

Aus den Fragestellungen wird bereits deutlich, dass wir den Begriff, Widerstand“weiter fassen wollen, nämlich über die juristisch-verfassungsrechtliche Begrifflichkeit des Widerstandsrechts hinaus, das nur unter ganz bestimmten Bedingungen eine Auflehnung gegen staatliche Gesetze und Institutionen gestattet, nämlich erst dann,

- wenn alle institutionellen Wege der Beschwerde oder des Rechtsmittels ausgeschöpft sind und

- überdies die verfassungsmäßige Grundordnung außer Kraft gesetzt wird oder droht, außer Kraft gesetzt zu werden.

Wir wollen - neben dem Widerstandsrecht - auch in einem umfassenderen Sinne über „politischen Widerstand“ sprechen,

- der neben und über die justiziellen Beschwerdewege hinaus tritt und

- bereits unterhalb der Schwelle einer akuten Bedrohung oder Beseitigung der Grundordnung eintreten kann.

Gemeinsam ist dem juristischen und dem politischen Begriff die begründete Vermutung des Widerstand Leistenden, dass fundamentale Prinzipien oder Lebensgrundlagen des Gemeinwesens durch staatliches Handeln/durch politische Entscheidungen gefährdet sind. Das kann die Klimakatastrophe im Bereich der Umweltpolitik oder der Verfall des Rechtsstaats im Bereich der Rechtspolitik sein.

Das Widerstandsrecht hat ganz zwangsläufig auch mit dem Thema Gewalt zu tun, weil das Recht auf Widerstand gegen staatliche Einrichtungen qua definitionem das staatliche Gewaltmonopol angreift, das ja das gesamte Rechtswesen durchzieht - und natürlich eine ganz wesentliche zivilisatorische Errungenschaft darstellt. Hinter diesem gleichsam geordneten Angriff auf das Gewaltmonopol - durch das Widerstandsrecht steht die Überlegung, dass auch der Staat selbst Unrecht tun kann, selbst wenn das auf der Grundlage von Gesetzen geschieht. Spätestens seit dem Nationalsozialismus ist diese Erkenntnis zum Allgemeingut geworden.

Peter-Alexis Albrecht wird einleitend den Weg rekonstruieren, der uns - nach seiner Auffassung - in eine unerträgliche Sicherheitsgesellschaft führt, und fragen, ob überhaupt und - gegebenenfalls - wie gegen eine solche Entwicklung Widerstand geleistet werden kann.

\section{B. Die Entwicklung der Erosionen zentraler Rechtsprinzipien (Peter-Alexis Albrecht)}

Die Entwicklung des Strafrechts als Eckpfeiler formeller sozialer Kontrolle in der Bundesrepublik Deutschland verlief in den letzten 50 Jahren vom

- sozial-integrativen Strafrecht der 60er und 70er Jahre (1.)

- über eine umfassende Präventionsorientierung in den 80er und 90er Jahren (2.)

- hin zu einer komplexen Sicherheitsgesellschaft des Grundrechteverzehrs seit der Jahrtausendwende (3.). 
Sollte es nicht möglich sein, diese Entwicklung durch Rechtsprinzipien auf rechtsstaatlichen Wegen aufzuhalten, wird sich unkontrollierter Widerstand auftun, wie ihn die Geschichte des Widerstands in vielerlei Formen belegt. Vor dem Hintergrund bedrohlicher, auch globaler Gewalteskalationen ist die Suche und Anwendung staatskritischer Absolutheitsregeln zur Stabilisierung von Menschen- und Grundrechten zwingend. Das ist die Hoffnung des freiheitlichen und sozialen Rechtsstaates. Diese Hoffnungslinie ist keine neue, aber gleichwohl höchst aktuell: Es geht dabei um den „Versuch, die Grenzen der Wirksamkeit des Staates zu bestimmen“- wie es Wilhelm von Humboldt schon 1792 unternahm -, und um die mutige und unabhängige Mitwirkung der Rechtsprechung, dem Staat absolute Grenzen klar und deutlich zu setzen. Wo könnte man das symbolischer aufzeigen als in diesem Historischen Plenarsaal des ehemaligen Reichsgerichts und heutigen Bundesverwaltungsgerichts: Der Ort, an dem einst die von Richtern judizierte Rückwirkung der Todesstrafe die Welt anschließend an den Abgrund geführt hat, der heute aber der Ort konkreter rechtsstaatlicher Grenzziehungen geworden ist, mittels derer staatliche Macht im Rechtsstaat gebunden wird.

\section{Das sozial-integrative Strafrecht des Wohlfahrtsstaates}

Der westdeutsche Wohlfahrtsstaat der 60er und 70er Jahre ist durch ökonomische Leitbilder, zentrale Kontrollziele, umfassende Transformationsprozesse sowie durch Bedingungen seiner Auflösung, den Abbau seines Idealbildes und einer Schwerpunktverlagerung sozialer Kontrolle gekennzeichnet. Im Einzelnen: ${ }^{5}$

- DER WOHLFAHRTSSTAAT in seiner keynesianischen Variante war an den Leitbildern der Vollbeschäftigung, der sozialen Rechtsteilhabe, des Massenkonsums und am Ideal der Sozialpartnerschaft orientiert.

- DER WOHLFAHRTSSTAAT stellt als zentrales Ziel sozialer Kontrolle auf die Integration des Einzelnen ab. Es ist eine Disziplinierung im Sinne kompensierenden sozialen Ausgleichs und sozialer Integration. Vorherrschend ist eine lösungsorientierte Behandlung sozialer Probleme.

- DER WOHLFAHRTSSTAAT erlebte zum Ende hin umfassende Transformationsprozesse, die zu einer hohen Flexibilisierung und Deregulierung der Produktions- und Arbeitsverhältnisse führten. Die Automatisierung setzte massenweise Arbeitsplätze frei. Die Arbeitsplatzsicherheit nahm ab und unsichere und ungeschützte Arbeitsverhältnisse nahmen in erheblichem Ausmaß zu. Eine sich abzeichnende Privatisierung staatlicher Aufgaben leitete den grundlegenden Umbau des Wohlfahrtsstaates ein. Kennzeichen dieses Prozesses waren eine stärkere Ausdifferenzierung der Sozialstruktur, zunehmende soziale Desintegration und starke gesellschaftliche Verunsicherungen.

- DER WOHLFAHRTSSTAAT erwies sich als recht kurze Phase in der Periode seiner eindeutig expansiven und intervenierenden Entwicklung mit dem Anspruch auf um-

5 Alle Literaturnachweise und Fundstellen befinden sich in Peter-Alexis Albrecht, Der Weg in die Sicherheitsgesellschaft, Berliner Wissenschaftsverlag, 2010: Zweiter Teil, Dritter Abschnitt (S. 141 ff.), Dritter Teil, Dritter Abschnitt (S. 523 ff) und Fünfter Teil, Dritter Abschnitt (S. 809 ff.). Weiterführend vgl. auch im Folgenden Tobias Singelnstein/Peer Stolle, Die Sicherheitsgesellschaft. Soziale Kontrolle im 21. Jahrhundert, 2. Auflage, 2008. 
fassende Gesellschaftssteuerung. Seine Defizite drückten sich vor allem darin aus, dass die realen Folgen der krisenhaften ökonomischen Entwicklung (Arbeitslosigkeit, Sozialhilfebedürftigkeit, Wohnungsnot) sozialpolitisch nicht mehr entsprechend abgefedert und kompensiert werden konnten.

- DER WOHLFAHRTSSTAAT wurde in seinem Idealbild infolge ökonomischer Umbrüche einer Staatlichkeit im Wandel und massiver sozialstruktureller Veränderungen in der postmodernen Gesellschaft umfassender staatlicher Regulierung erheblich in Frage gestellt. Die materiale Ausprägung des Sozial- und Rechtsstaates blieb auf halbem Wege stehen.

- DER WOHLFAHRTSSTAAT führte bei seiner Auflösung zu einer Verlagerung des Schwerpunktes und zu einem Wandel der Strukturen sozialer Kontrolle.

\section{Das Strafrecht des Präventionsstaates}

Das Profil des Präventionsstaates zeigt sich in der Entwicklung von der Integration zur Verwaltung von Abweichung, in der Umorientierung vom Schaden zum Risiko, im Wechsel empirischer Erprobung zu normativer Erwartung und generell in Eingriffsvorverlagerungen. In der Informalisierung des Rechts schlägt die Stunde der Exekutive, das Kriminaljustizsystem wird als gesellschaftliche Reparaturanstalt missverstanden. Gesetzt wird auf Symbolik anstelle materialer Gerechtigkeit, auf Selektivität anstelle Gleichheit. Vom Bürger wird der Feind separiert, die Problemlösung weicht der Problemverwaltung (Exklusionen). Die Pathologisierung verdrängt das Individuelle, das Individuum verliert sich in der Bekämpfung von Problemlagen. Menschenrechte versinken in präventiver Grenzauflösung. Im Einzelnen:

- IM PRÄVENTIONSSTRAFRECHT verlagert sich der Schwerpunkt sozialer Kontrolle von der Integration durch Resozialisierung zur Verwaltung von Abweichung. An die Stelle des allgemeinen Resozialisierungsversprechens tritt nunmehr das Versprechen individueller Sicherheit. Der Sozialstaat wird tendenziell verlassen. Der Obrigkeitsstaat kommt erneut in den Fokus der Kriminalpolitik.

- DAS PRÄVENTIONSSTRAFRECHT ist ein Strafrecht der Risikovorsorge unter offen erklärter Zuschreibung. Es fordert Gefährdungsdelikte, wobei die Gefahr für den Eingriff des Rechts bereits ausreicht, nicht mehr der konkrete Verdacht. Die Kontrolltechniken sind damit nicht mehr individual-zentriert, sondern risiko-orientiert.

- DAS PRÄVENTIONSSTRAFRECHT favorisiert die negative Spezialprävention. Der Gefangene kann der Aussetzung des Strafrestes nur noch dann entgegen sehen, wenn dies unter der Berücksichtigung der Sicherheitsinteressen der Allgemeinheit vom Richter verantwortet werden kann. Hierbei handelt es sich um einen kriminalpolitischen Wertungsschritt, der sich von der ursprünglichen Erprobungsklausel durch eine kriminalpolitische Wertungsklausel (vgl. § 57 Abs. 1 Nr. 2 StGB) signifikant unterscheidet.

- DAS PRÄVENTIONSSTRAFRECHT ist Vorfeldbekämpfung. Die kennzeichnenden Mechanismen sozialer Kontrolle sind Vorverlagerung und Ausweitung staatlicher Zugriffe. Grenzen zwischen Polizei und Strafrecht verwischen. Im Präventionsstrafrecht findet seit den 80er Jahren im Bereich der Polizei eine Wende zur operativen Prävention statt. 
- DAS PRÄVENTIONSSTRAFRECHT reagiert aufgrund der Überfrachtung der Strafjustiz mit einer Fülle von überlastenden Aufgaben, d.h. mit präventiven Überforderungen, politischen Aufladungen und instrumentellen Überlastungen mit bürokratischem Kollaps. Rechtssoziologen haben diese Prozesse beobachtet und als Prozess bürokratischer Verflachung, als ,administrative Rationalisierung“ beschrieben. Typischer Beleg aus dem Verfahrensrecht ist die zunehmende Bewältigung komplexer Sachverhalte in Strafverfahren mit Hilfe von Opportunitätsnormen oder informellen Absprachen. Hierbei wird der Anwendungsbereich des Rechts ungleich umgesetzt, statt allgemeingültige und gleiche Eingriffsvoraussetzungen $\mathrm{zu}$ formulieren. Das Strafrecht wird informalisiert und öffnet sich zum exekutivischen Recht, von der Kontrolle durch eine unabhängige Judikative weit entfernt. Zunehmend verliert das rechtsstaatliche Strafrecht seine Fähigkeit, staatliche Macht zu binden.

- DAS PRÄVENTIONSSTRAFRECHT zerbricht mit seinen Überforderungen das rechtsstaatliche Kriminaljustizsystem. Der Zugriff des rechtsstaatlichen Strafrechts muss sich auf zentrales Unrecht konzentrieren, muss es kenntlich und öffentlich machen - in rechtsstaatlicher und justizförmiger Vorbildlichkeit. Zieht man diese Konsequenzen nicht, drohen Verformung und Zerstörung des zerbrechlichen rechtsstaatlichen Strafrechts und seiner Institutionen. Dann droht der Umschlag in staatlich produziertes Unrecht. Nur in seinem beschränkten, strikt rechtsstaatlichen Zugriff entfaltet das Strafrecht seine freiheitsschützende Wirkung.

- DAS PRÄVENTIONSSTRAFRECHT zerstört mit seinen übersteigerten Systemschutzanforderungen das rechtsstaatliche Strafrecht, da systemisches Steuerungsversagen prinzipiell nicht individualisierbar, weil nicht schuldkompatibel ist. Versucht man dies dennoch - wie in der Strafjustiz jenseits der informalisierenden Verfahrenseinstellungen selektiv noch anzutreffen -, bleibt die Gerechtigkeit in aller Regel auf der Strecke, trotz des verschämten Ziehens aller Strafmilderungsregister. Belege sind die jahrelangen Wirtschafts-, Umwelt- und Politprozesse, die - statistisch signifikant - zumeist nur Geld- und Bewährungsstrafen nach sich ziehen. Das ist symbolisches Strafrecht und symbolische Strafjustiz.

- DAS PRÄVENTIONSSTRAFRECHT missbraucht über die exemplarischen Aktivitätsnachweise die strafjustitielle Praxis. Durch die symbolischen Strafrechtsanforderungen bleibt nicht nur das rechtsstaatliche Strafrecht auf der Strecke, das ungleich, selektiv und unter Verletzung des verfassungsrechtlichen Schuldprinzips Anwendung findet. Vor allem bleiben auch die Problemlösungskapazitäten einer Gesellschaft unentwickelt, also die Fähigkeit zu einer freiheitlichen, eigenverantwortlichen Krisenund Problemlösung.

- IM PRÄVENTIONSSTRAFRECHT stellt soziale Kontrolle nicht mehr auf den individuellen Hilfsbedürftigen ab, im Mittelpunkt steht der ,gefährliche Andere` - der Feind! Soziale Absicherung im Sinne des Wohlfahrtsstaates tritt zurück. Es werden Abschottungsmechanismen und Konstruktionen eines Feindstrafrechts eingeführt.

- DAS PRÄVENTIONSSTRAFRECHT entfernt sich vom Rehabilitationsideal und sucht anstelle dessen eine punitive Orientierung an Opferinteressen, Sicherheitsidealen und Risikoorientierungen. Es verlässt die Ebene der individuellen Moralisierung und betritt die Bühne der Pathologisierung von Individuum und Gesellschaft.

- DAS PRÄVENTIONSSTRAFRECHT sieht jeden als potentiellen Normbrecher an. Die soziale Kontrolle wandelt sich vom Blick aufs Individuum hin zu Strukturen, Lagen, 
Orten, die Devianz ermöglichen. Probleme sind nicht mehr beim Täter persönlich zu verorten, sondern die Normabweichung bekämpft flächendeckend soziale Problemlagen. Das Präventionsstrafrecht sieht nicht mehr Armut als zu beseitigenden Zustand an, sondern die Armen sollen im Sinne einer Problemlösung weggeschlossen werden.

- IM PRÄVENTIONSSTRAFRECHT finden Reduktionen der materialen Komponente der Grundrechte, also Eingriffe in den absoluten Kern der Menschenrechte, zugunsten einer systemisch-präventiven Grenzauflösung statt.

\section{Die Sicherheitsgesellschaft}

Das Präventionsstrafrecht war nicht das Ende einer Entwicklung. Mit dem 11. September 2001 vollzog sich nicht nur eine Verschärfung, vielmehr tat sich ein qualitativ neues Ziel für die Gesellschaft insgesamt auf. Der Sicherheit wurde die Priorität vor der Freiheit eingeräumt. Der Weg in die Sicherheitsgesellschaft ist Ausdruck von Transformationsprozessen im Rahmen von massiven Strukturproblemen der postmodernen Weltgesellschaft. Welcher Art sind diese Strukturprobleme? Offenbar sind es ökonomische Umwälzungen und damit einhergehende Verschiebungen in der Funktion des Staates, worauf der sozio-kulturelle Überbau reagiert. Im Einzelnen:

\section{Dominanz der ökonomischen Logik}

Die ökonomischen Veränderungen werden ausgelöst durch zwei ineinander greifende Prozesse: Die technische Entwicklung zur weitgehend automatisierten, mit den Mitteln der Informationstechnologie gesteuerten Produktion führt zu einer dramatischen, dauerhaften Verringerung des Arbeitskräftebedarfs. Gleichzeitig führt die weltweite Arbeitsteilung zur Auslagerung lohnintensiver Produktion in Länder niedrigen Lebensstandards und damit korrespondierend: niedriger Löhne, was im Gegenzug in den hoch entwickelten Industriegesellschaften zu einer weiter sinkenden Nachfrage nach - lohnintensiver - Arbeit führt. Durch die globale Konkurrenzsituation in zusammenwachsenden Märkten schaukelt sich dieser Prozess weiter hoch: Kein Unternehmen kann sich der ökonomischen Logik entziehen.

\section{Rückzug des Staates}

Der Staat erweist sich vor dem Hintergrund dieser Entwicklung mit seinem nationalstaatlich gebundenen Eingriffsinstrumentarium zunehmend als untaugliches Steuerungsorgan für die Produktions- und Arbeitsverhältnisse. Auf die globale Lohnkonkurrenz wird mit der Absenkung des eigenen Lohnniveaus geantwortet: Die Voraussetzungen für staatliche Transferleistungen werden erhöht, der Niedriglohnsektor wird ausgeweitet. Aber auch bezogen auf nationale Wirtschaftsmärkte, die bislang Gegenstand staatlicher Intervention waren, gewinnen Konzepte die Oberhand, wonach staatliche Eingriffe einen effizienten Markt und freie Wirtschaftsteilnehmer zu behindern scheinen. Staatseigene oder staatlich kontrollierte Wirtschaftsbereiche werden durch Privatisierung dem Markt überantwortet ebenso wie vormals staatlich organisierte Vor- 
sorge- oder Sozialleistungen mit privatwirtschaftlichen Komponenten durchsetzt werden.

\section{Entstehung des Prekariats}

Durch diese Entwicklungen verringert sich der Bedarf an lebenslangen, festen, normalbezahlten Arbeitsverhältnissen zugunsten zeitweiliger, unsicherer und unterbezahlter Beschäftigung. Der Arbeitsmarkt kann seine gesellschaftliche Kernfunktion, nämlich den Lebensunterhalt für die arbeitswilligen Gesellschaftmitglieder zu ermöglichen, tendenziell nicht mehr erfüllen. Arbeitslosigkeit wird von einem temporären Ereignis zur Lebenslage, es entstehen ,prekäre“ Lebensverhältnisse. Im sozio-kulturellen Gesellschaftsbereich zeigen sich die ökonomischen und politischen Veränderungen als Abbau der bis dahin vorherrschenden Medien sozialer Integration wie der Kleinfamilie oder sozialer Beziehungsgeflechte, die der ökonomisch verlangten Mobilität geopfert werden. Der Einzelne sieht sich auf sich selbst zurückgeworfen, das soziale Sicherheitsnetz zerfällt. Vorsorge für Arbeitslosigkeit oder das Alter wird zu einer Zeit als individuelle Problemlage rekonstruiert, wo die gesellschaftliche Solidarität eigentlich besonders gefragt wäre, geht doch die persönliche Lebensgewissheit vor dem Hintergrund unsicherer Arbeitsverhältnisse zunehmend verloren. Die gesamte Gesellschaft umfassende Deutungskonzepte in kultureller, wirtschaftlicher oder religiöser Hinsicht zerfallen; es entsteht ein Nebeneinander subkultureller Orientierungen. Und im Zerfall allgemeingültiger Erklärungsmodelle wachsen die Möglichkeiten für fundamentalistische Weltdeutungen oder Religionen in Nischengesellschaften. Nun ist der Boden bereitet, auf dem „Sicherheit" als Weltformel gedeihen kann.

\section{9/11: Nicht Grund, nur Anlass}

Behält man die skizzierten Transformationsprozesse - gerade auch globaler Art - im Blick, bekommen die Anschläge auf die amerikanischen Wirtschafts- und Dominanzsymbole eine andere Dimension. Dieser 11. September 2001 war nicht nur für die Opfer in New York und für den Weltfrieden eine Katastrophe, sondern auch für das rechtsstaatliche Strafrecht weltweit ein schwarzer Tag. Man hätte den USA Besonnenheit und kühlen Kopf bei der Ursachenanalyse gewünscht. Das Gegenteil trat ein. Das Recht des Stärkeren galt von nun an umso mehr, die Stärke des Rechts erodierte - weltweit. Auflösungsprozesse erfassten die Menschenrechte, die Grundlagen rechtsstaatlicher Strafgesetzlichkeit und rechtsstaatlicher Freiheitsentziehung. Aber auch kritische Gesellschaftswissenschaften zogen sich zurück angesichts einer vom Konsens getragenen Sicherheitsgesellschaft, die mittels von Mehrheiten gewollten Rechtsabbaus das Ausmaß ihrer Verunsicherung einzugrenzen versucht - vergeblich. Die Politik des Westens suchte nach Antworten auf die Herausforderungen einer kollabierenden Welt. Die Strukturprobleme einer Weltgesellschaft, die verständliche Sicherheitsbedürfnisse der Menschen entfachen, die Anforderungen der Industriegesellschaften, die als Risikogesellschaften bezeichnet werden und Eruptionen der Gewalt sowie suizidale Selbstaufgaben fördern eine - leider zumeist unreflektierte - Gesetzgebungsmaschine, die immer nur nachklappt, weltweit. Bei all dieser an Fatalität grenzenden Rückbezogenheit wer- 
den die Grundlagen dessen, was es zu schützen gilt, vernichtet. Wenn Bedrohungslagen die Freiheit des Menschen ersticken, ist eine in Aussicht gestellte Sicherheit nur als totale Staatssicherheit möglich, ist dann aber Staatsterrorismus.

\section{Präventiv-Folter: Der Weg in den Staatsterrorismus}

Das Opfer der Gesellschaften liegt aber nicht nur im Verlust zahlreicher Menschenleben. Das gesellschaftliche Opfer ist die Aufgabe des Rechtsstaates und der Menschenrechte. Die aufziehende - juristisch ernsthafte - Folterdebatte in Deutschland nach dem 11. September 2001 war der bislang radikalste Umschlagspunkt der Rechtspolitik vorerst im strafrechtlichen Fachschrifttum: Ein rechtswissenschaftlicher Diskurs, der sich von den Menschenrechten abwandte, um die existentielle Angst vor diffuser Bedrohung und Risiken unter Preisgabe menschlicher Würde abzubauen. Die juristische Folterdebatte, also Folter als Mittel der Innen- und Rechtspolitik diskussionsfähig zu machen, ist ein qualitatives Novum, das selbst in den Debatten zum Präventionsstaat noch nicht ernsthaft Niederschlag gefunden hatte. Bis 2001 ging man ganz überwiegend davon aus, dass nach dem Staatsterrorismus des NS-Regimes dieses Thema für deutsche Juristen für immer obsolet sein würde. So sah es auch Amnesty International in einem Memorandum, Nein zur Folter. Ja zum Rechtsstaat 'aus dem Jahr 2005: „In Deutschland leitet das Folterverbot bislang seine Legitimität aus dem kollektiven Entsetzen über die Barbareien des Nationalsozialismus ab. Der Verweis auf das historische Unrecht, das noch kein Menschenalter zurückliegt, reicht offenbar nicht aus, zu begründen, warum Folter absolut verboten ist und weiterhin verboten bleiben muss. “ Amnesty International sah sich gezwungen öffentlich aufzurufen, dass sich Bürgerinnen und Bürger über die „Bedeutung der grundlegenden demokratischen Werte unserer Gesellschaftsordnung erneut vergewissern und auch in diesem Zeitalter vielfältiger und komplexer Bedrohungen verteidigen“. Damit war die Debatte aus dem juristischen Fachdiskurs herausgelöst und einer breiten Öffentlichkeit überantwortet. Skandalisiert wurden die Relativierungstendenzen gegenüber dem absoluten Folterverbot. Erinnert wurde von Amnesty International an das uneingeschränkte Folterverbot im Völkerrecht. Die Diskussion um die Garantie der Unantastbarkeit der Menschenwürde wurde angestoßen, an die Lehren aus der deutschen Geschichte wurde erinnert und auf die ,Gefahren des Präventionsstaates" wurde sozialwissenschaftlich informiert verwiesen.

\section{Juristische Argumentationslinien „Pro Folter“: Umsetzung noch offen}

Dem gegenüber stellt die juristische Fachdebatte die internationale Verankerung des Folterverbots in Frage. Anwendungslegitimationen werden aus dem Verfassungsrecht hergeleitet, in regionalem Polizeirecht werden Ermächtigungen erblickt und strafrechtliche Rechtfertigungs- und Notstandsvorschriften werden für Folter anwendungsoffen interpretiert. Schließlich wird die Legitimität von Folter auch durch Analogie zum finalen polizeilichen Rettungsschuss hergeleitet. Diese normative Debatte sucht Lücken und Einbruchstellen in strafrechtsdogmatischen Konstruktionen. Die juristischen und politischen Argumentationslinien sind vorgezeichnet. Lediglich der Zeitpunkt der Umsetzung ist noch offen. 


\section{Tribut der Sicherheitsgesellschaft}

Folter - in den USA seit 9/11 praktizierte Vernehmungsmethode - ist in Deutschland (noch) kein anerkanntes Mittel des Polizei- oder Strafrechts. Der Prozess der Rechtserosion nagt gleichwohl an dieser rechtsstaatlichen Bastion. Die Sicherheitsgesellschaft fordert ihre grundrechtsverzehrenden Tribute im Kampf gegen die allgemeine Verunsicherung. Und das, ohne bisherige Angriffe auf Ziele in Deutschland. Man mag sich kaum ausdenken, welche Fahrt dieser Prozess aufnehmen würde, wenn es Opfer zu beklagen gäbe.

\section{Weimarer Analogie: Vernichtung ,lebensunwerten“ Lebens}

Für den Fall terroristisch motivierter Gewaltanschläge in Deutschland sind die Schubladen der Inneren Sicherheit nur noch zu öffnen. Die Situation ähnelt in fataler Weise der von Naucke nachgezeichneten Debatte über die „Vernichtung lebensunwerten Lebens" bereits in den frühen Jahren der Weimarer Republik. Der Psychiater Hoche und der Strafrechtslehrer Binding - zwei höchst angesehene Repräsentanten ihrer Zünfte hatten minutiös den späteren Mordkampagnen des Dritten Reiches eine scheinbare Legitimationsgrundlagen geschaffen, ohne jeglichen Protest, noch nicht einmal aus der Fachöffentlichkeit. Dass es in der Ära der Weimarer Republik niemand gewusst haben kann, dass der Staat alsbald kranke Menschen ermorden würde, kann nach diesen Legitimationsversuchen des juristischen Grauens niemand mehr behaupten. Mord an Kranken war strafrechtsdogmatisch als ,gesundheitshygienische Maßnahme“ definiert und umgedeutet worden - eine vorweggenommene Freisprechung der (späteren) Mordtäter.

\section{Zusammenfassend: Das prinzipiell Neueste an der Sicherheitsgesellschaft}

Die Sicherheitsgesellschaft ist gekennzeichnet von einem Teufelskreis von Sicherheit und Unsicherheit, sie ist getragen von der existenziellen Angst vor Bedrohungen und Risiken. Sicherheit führt zu einer totalen Durchdringung der Gesellschaft und die Sicherheitsgesellschaft ist der Zustimmung der Mehrheitsgesellschaft sicher. Der Preis für angebliche Gewissheitsverluste ist die Konsensualisierung des Kriminaljustizsystems, das Ziel ist ,Sicherheit', die gefährliche Totalität der normativen Sicherheitsproduktion indiziert eine staatliche und gesellschaftliche Sicherheitsparanoia.

- IN DER SICHERHEITSGESELLSCHAFT tritt ein gesteigertes Bedürfnis nach Sicherheit auf. Die permanente Suche nach Schutz reklamiert den schützenden Staat. Weil aber ein dauerhafter Schutz von diesem deregulierten Staat gar nicht zu leisten ist, wird neue Verunsicherung produziert. Dies erfordert nur noch „,symbolisches Regieren“, ein „Regieren aus Distanz“.

- IN DER SICHERHEITSGESELLSCHAFT führen auch Egoismus, Marktliberalität und Deregulierung zu einer Form umfassender Verunsicherung. Der Wunsch nach persönlicher Sicherheit vor Bedrohungen und Risiken nimmt zu. Neu ist die verbreitete Angst, die soziale Existenz zu verlieren. 
- IN DER SICHERHEITSGESELLSCHAFT ist die Dialektik von Privatisierung und Entgrenzung staatlicher Macht eine neue staatliche Kontrollform mit einer Tendenz zur totalen Durchdringung der Gesellschaft. ,Effizienz', ,Transparenz' und ,Qualität sind moderne exekutive Anforderungen für das Funktionieren einer Sicherheitsmaschinerie, wobei die entscheidende Frage nach Recht und Unrecht völlig in den Hintergrund tritt. Das ist ein Indiz für die Dominanz des Prinzips der Ökonomie vor dem Prinzip des Rechts.

- IN DER SICHERHEITSGESELLSCHAFT ist als neues soziales Phänomen für dessen Legitimation die Zustimmung der Rechtsunterworfenen charakteristisch. Aus Furcht vor komplexer Unsicherheit wird die Sicherheit des Rechtsschutzes von den zu Schützenden freiwillig aufgegeben.

- IN DER SICHERHEITSGESELLSCHAFT finden eine Kontrollvorverlagerung und eine Ausweitung informeller Kontrolle statt. Die normativen Voraussetzungen hierfür werden durch das Prinzip einer Konsensualisierung eingerichtet. Gesellschaftliche Gewissheitsverluste - ein angebliches Charakteristikum der Postmoderne - werden als fragwürdige Legitimationsgrundlage für die Normierung von Konsensualisierung im Strafrecht angeboten.

- IN DER SICHERHEITSGESELLSCHAFT wird ,Sicherheit" somit zum exklusiven Ziel sozialer Kontrolle. Es ist nicht jede einzelne Neuregelung, die isoliert betrachtet als vertretbar angesehen werden könnte. Vielmehr ist es die gefährliche Totalität, die sich aus der Summe der zahlreichen gesetzlichen Bestimmungen der letzten zehn bis zwanzig Jahre, die angeblich der inneren Sicherheit dienen sollten, ergibt.

Abschließend: Gelingt es nicht, die Irrationalität der Sicherheit - dieses wuchernde Krebsgeschwür im Recht - aufzulösen, droht ein Chaos ungezügelter Gewalt, wie es uns aus jüngster Vergangenheit und Gegenwart deutlich vor Augen stehen sollte. Das Glück der gewaltlosen deutschen Einigungsgeschichte von 1989 dürfte dabei Ausnahme sein. Bei der Positivierung des Widerstandsrechts in Art. 20 Abs. 4 des Grundgesetzes sind seine Väter und Mütter von Gewaltlosigkeit jenseits des Rechtsschutzes jedenfalls nicht ausgegangen. Gewalt zum Schutz der Verfassung ist - ,wenn andere Abhilfe nicht möglich ist" - ultima ratio des verfassungstreuen Bürgers. ${ }^{6}$ Aber wo führt uns diese Gewalt hin? Wir wollen dazu andere befragen, die mehr Kompetenz und eigene Erfahrungen mit aktivem Widerstand haben.

\section{Zur Geschichte paradigmatischer Widerstandsformen}

\section{Georg Elser: Widerstand in der NS-Zeit (Ulrich Renz) ${ }^{7}$}

Das Attentat von Georg Elser liefert meines Erachtens keine Lehren für die Gegenwart in der Bundesrepublik Deutschland. Elser kämpfte gegen ein beispielloses Gewaltregime. Und er stimmte mit der Mehrzahl der aktiven Widerständler darin überein, dass die Lösung des Problems nur in der Beseitigung von Adolf Hitler liegen könne. In dieser Hinsicht ist Elser kein Vorbild für Zeiten, in denen andere Möglichkeiten des Widerstandes gegen staatliches Handeln möglich sind. Allerdings werde ich am Ende meiner

6 Roman Herzog in Maunz-Dürig, Komm. z. GG, Art. 20 Rn. IX 34.

7 Vgl. hierzu Ulrich Renz, Georg Elser - Ein Meister der Tat, Leinfelden-Echterdingen 2009. 
kurzen Ausführungen eine Frau zitieren, die von einem Vermächtnis Elsers auch für unsere Zeit spricht.

Um den Hintergrund kurz zu erklären: Der Schreiner Georg Elser aus Königsbronn auf der Schwäbischen Alb baute im Jahre 1939 in über 30 Nächten eine Bombe in eine Säule im Saal des Bürgerbräukellers in München ein, in dem Hitler jeweils am 8. November in Erinnerung an den Putschversuch von 1923 sprach. Die Sprengladung, die Elser beharrlich „Apparat“ nannte, explodierte auch zur genau vorberechneten Zeit. Acht Menschen starben, über 60 wurden verletzt. Doch Hitler hatte völlig überraschend 13 Minuten zuvor den Saal verlassen, um den Sonderzug zu erreichen, der ihn zur weiteren Vorbereitung des Feldzuges gegen Frankreich nach Berlin bringen sollte. Ein Flug war wegen Nebels nicht möglich. Elser wurde auf der Flucht an der Schweizer Grenze festgenommen, gestand wenige Tage später, saß bis Kriegsende in völliger Isolation im KZ und wurde am 9. April 1945 in Dachau erschossen.

Aufschluss über Elsers Tat und seine Motive gibt weitgehend das Protokoll seiner Vernehmung durch die Gestapo, das in den 1960er Jahren vom Historiker Lothar Gruchmann entdeckt und 1970 veröffentlicht wurde. Es ist natürlich mit etwas Vorsicht zu betrachten, denn es ist, wie gesagt, das Werk der Geheimen Staatspolizei. Doch auch angesichts dieser Einschränkung lässt es klar erkennen, was Elser umtrieb und wie er seine Tat rechtfertigte. Deutlich benennt er seine Gründe, auch wenn er dies, seiner Art entsprechend, eher schlicht formuliert. Er sagte beispielsweise: „Nach meiner Ansicht haben sich die Verhältnisse in der Arbeiterschaft nach der nationalen Revolution in verschiedener Hinsicht verschlechtert. So zum Beispiel habe ich festgestellt, dass die Löhne niedriger und die Abzüge höher wurden.“

Georg Elser war ein Mensch mit einem ausgeprägten Gerechtigkeitssinn. Fühlte er sich etwa an seinem Arbeitsplatz ungerecht behandelt, dann ging er. Sein Verständnis von Politik war stark durch sein persönliches Freiheits- und Unabhängigkeitsstreben geprägt. Es ist verbürgt, dass er den Nationalsozialismus von Anfang an ablehnte und dies auch zeigte: Er verweigerte den „Deutschen Gruß“, von Aufmärschen und Fahnen wandte er sich ab, bei der Übertragung von Reden von Hitler und seinen Paladinen verließ er den Raum. Seine ganze Persönlichkeit, sein Charakter vertrugen sich nicht mit einer totalitären Herrschaft.

Im Verhör berief er sich auf die Verschlechterung der Lage der Arbeiter, wobei er genau vorrechnete, wie sich dies in Mark und Pfennig auswirke. Er erwähnte die Einschränkung von Freiheitsrechten, etwa bei der Wahl des Arbeitsplatzes oder in religiösen Dingen. Und er sagte, der Arbeiter ,ist heute durch die HJ nicht mehr Herr seiner Kinder."

Diese Überlegungen beschäftigten ihn schon vor 1938, dem Jahr, in dem er nach eigenen Angaben den Entschluss zur Tat fasste. Zuletzt aber wurde für ihn die Furcht vor einem Krieg die größte Antriebskraft. Er rechnete mit dem Krieg und ließ sich auch durch das Münchener Abkommen von 1938 nicht beirren - ganz im Gegensatz zur weit verbreiteten Ansicht, damit sei der Friede gesichert. Er sagte nach seiner Verhaftung im November 1939: „Ich war bereits voriges Jahr um diese Zeit der Überzeugung, dass es bei dem Münchener Abkommen nicht bleibt, dass Deutschland anderen Ländern gegenüber noch weitere Forderungen stellen und sich andere Länder einverleiben wird und dass deshalb ein Krieg unvermeidlich ist.“ Er sagte: „Ich wollte ja auch durch meine Tat ein noch größeres Blutvergießen verhindern." 
Und Elser zog den Schluss: „Die von mir angestellten Betrachtungen zeitigten das Ergebnis, dass die Verhältnisse in Deutschland nur durch die Beseitigung der augenblicklichen Führung geändert werden könnten." Er nannte dabei Hitler, Göring und Goebbels und hegte die Erwartung, dass ihnen Männer in der Regierung folgen würden, „die an das Ausland keine untragbaren Forderungen stellen und die für eine Besserung der sozialen Verhältnisse der Arbeiterschaft Sorge tragen werden“.

Soweit der Attentäter und seine Motive. Um es noch einmal zu betonen: Es ging um die Beseitigung Hitlers. Oder um den ehemaligen hessischen Generalstaatsanwalt Fritz Bauer zu zitieren, der 1962 in einer Betrachtung über den „Widerstand des kleinen Mannes“ schrieb: „Jeder, ob groß oder klein, ist berechtigt, einen Mörder an der Fortsetzung seiner Verbrechen zu hindern.“ Bauer erklärte auch: „Passiver Widerstand gegenüber verbrecherischen Gesetzen, Befehlen, Handlungen eines Staates ist Recht und Pflicht eines Jeden. Zu aktivem Widerstand gegenüber Verbrechen ist niemand verpflichtet, wohl aber berechtigt, wobei die Grundsätze jeden Notwehrrechts gelten, dass die Verteidigung dem jeweiligen Angriff angemessen sein muss. Die Frage der Angemessenheit spielt aber in der Auseinandersetzung mit den Verbrechen des ,Dritten Reiches' keine Rolle.“

Damit ist die absolute Ausnahmesituation umschrieben, in der sich der aktive Widerstand gegen die Nationalsozialisten befand. Dieser Widerstand konnte, musste aber nicht ein Konzept für die Zeit nach der Beseitigung Hitlers bereithalten. Elser hatte es nicht. Aber es trifft auch nicht zu, dass er sich keine Gedanken über die Folgen gemacht hätte. Er vertraute darauf, er konnte darauf vertrauen, dass die neue Führung oder Regierung weniger radikal vorgehen würde. Wenn sein Anschlag Erfolg gehabt hätte, hätte er womöglich 50 Millionen Menschen das Leben gerettet.

Georg Elser verkörpert also ganz exemplarisch das Widerstandsrecht, das Jedermann zusteht. Ich betone im Sinne von Fritz Bauer: Jedermann. In den früheren Jahren der Bundesrepublik Deutschland hat es nicht zuletzt von Seiten der Justiz, die ja mit alten Nazis durchsetzt, wenn nicht gar von ihnen beherrscht war, Versuche gegeben, dieses Recht eingeschränkt zu betrachten, es nur Angehörigen sogenannter Eliten zuzugestehen. Es war die Rede davon, der Bundesgerichtshof habe mit seiner Rechtsprechung „Widerstandsprivilegien“ geschaffen. Diese Zeiten sind spätestens seit den 1990er Jahren vorbei. Lothar Gruchmann schreibt dazu: „Auch die Justiz unseres freiheitlichendemokratischen Rechtsstaates, die auf die Achtung der Würde jedes einzelnen Menschen und den Schutz der ihm angeborenen Recht angelegt ist, beurteilt heute die Widerstandstat des damaligen Einzeltäters gegen eine menschenverachtende Staatsführung nicht mehr nur aus dem positivistischen Blickwinkel der Verletzung einzelner Strafnormen, sondern des übergesetzlichen Notstandes. Sie beschränkt die Ausübung dieses als legitim anerkannten Widerstandsaktes auch nicht mehr auf einem begrenzten Personenkreis, der im Staatsgefüge besondere Verantwortung trägt, sondern billigt sie in der damaligen Situation jedem einzelnen zu, den das Gewissen nach ernsthafter Prüfung zum Handeln trieb.“

Noch ein Gesichtspunkt scheint mir wichtig. Ein Umstand, der mit dafür gesorgt hat, dass Georg Elser nach dem Krieg für sehr lange Jahre die Anerkennung verweigert wurde. Denn natürlich war er ein gewaltiger Störenfried im Heer der Mitbürger, die sich in der Ausrede einzurichten versuchten, dass Widerstand, zumal des Einzelnen, nicht möglich gewesen sei. Die Historikerin Benigna Schönhagen hat es in einem Bericht 
über den öffentlichen Umgang mit dem Widerstand gegen das NS-System in Tübingen nach 1945 so formuliert: „Denn je nachdem, ob der Widerstand verschwiegen, zu heroischen Tat einzelner monumentalisiert oder ob die vielfältigen Formen alltäglicher Verweigerung und couragierter Resistenz betrachtet werden, immer bedeutet die Auseinandersetzung mit dem Widerstand auch eine Einordnung des Verhaltens der Mehrheit im Faschismus."

Jutta Limbach, die ehemalige Präsidentin des Bundesverfassungsgerichts, hat vor geraumer Zeit doch den Bogen von der Tat Georg Elsers zur Gegenwart geschlagen. Zu einer Veranstaltung in Bremen erklärte sie in einer Betrachtung über Elser und das legalisierte Widerstandsrecht: „Nur sehr allmählich hat sich - mit dem Wandel von der Untertanen- zu einer Staatsbürgerkultur - in der Bundesrepublik ein Umdenken angebahnt. Seit den neunziger Jahren etwa sind wir dabei, nicht nur dem ,kleinen“ Widerstand, sondern auch dem aufbegehrenden Mann aus dem Volk wie Georg Elser Gerechtigkeit widerfahren zu lassen. Mit der Elser-Woche anlässlich des 100. Geburtstages von Georg Elser gibt die Stadt Bremen ein Signal: Sie ermahnt uns alle, unsere staatsbürgerlichen Rechte aktiv wahrzunehmen und durch unseren Widerspruchsgeist Eingriffe in Verfassungsrechte abzuwehren, auf dass wir staatlichen Machtmissbrauch nicht erst dann abzuwehren versuchen, wenn es zu spät ist. Das ist das Vermächtnis von Menschen, die wie Georg Elser gegen das nationalsozialistische Regime aufbegehrt haben. Die Bereitschaft zu steter Wachsamkeit. Diese ist der Preis der Freiheit und einer zivilen Gesellschaft.“

\section{Widerstandsformen in der DDR (Hans Misselwitz) ${ }^{8}$}

Was ist zu tun, wenn der hier vorgezeichnete, Weg in die Sicherheitsgesellschaft“"weder politisch noch juristisch abgewendet werden kann, wenn die Erosion des Rechtsstaats fortschreitet und die grundlegenden Freiheitsrechte beseitigt werden?

Hier schließen sich eine ganze Reihe von Fragen an: Von verantwortlicher Seite dürfte der Analyse ,Sicherheitsgesellschaft'- ganz sicher - widersprochen werden. Unabhängig davon: Wer entscheidet, ob das Recht auf Widerstand, das nach Art. 20 Abs. 4 des Grundgesetzes ,,alle Deutschen“ haben, wenn die von der Verfassung garantierte Ordnung in Gefahr und ,,andere Abhilfe nicht möglich ist", schon anwendbar ist? Sollte das etwa das Bundesverfassungsgericht selbst entscheiden? Und generell: Was sind Widerstandsformen noch vor jeder Erwägung von gewaltsamen Aktionen gegen Personen oder Institutionen?

Ich bin gebeten worden, der Frage nachzugehen, ob die Erfahrungen der Bürgerrechtsbewegung in der DDR und deren Beitrag für die gewaltfreie Überwindung der SED-Diktatur etwa ein Beispiel, ein Modell für einen Ausweg, also für einen Weg aus der Sicherheitsgesellschaft sein könnten.

Das erscheint allgemein abwegig. Die Unterschiede zwischen den Verhältnissen in der DDR und der Bundesrepublik liegen auch dann auf der Hand, wenn sich beim näheren Vergleich von sicherheits- und geheimdienstlichen Praktiken bedenkliche Annä-

8 Hans Misselwitz, Nicht länger mit dem Gesicht nach Westen. Das neue Selbstbewußtsein der Ostdeutschen, Bonn 1996. 
herungen zeigen lassen. Prinzipiell verschieden sind schon einmal die Verfassungsrealitäten. Die DDR-Verfassung erklärte den Staat als Diktatur, der Herrschaft einer Partei unterworfen. Gewaltenteilung gab es nicht, von Presse, Versammlungs- und Organisationsfreiheit konnte keine Rede sein, individuelle und politische Freiheiten stellte sie unter Gesetzesvorbehalt. Das heißt aber: Was das Widerstandsrecht nach Art. 20 Abs. 4 GG voraussetzt - die Gefährdung der demokratischen Ordnung - war in der DDR nicht vorhanden. Nicht die Verteidigung der Verfassung, sondern der Kampf um eine demokratische Verfassung stand auf der Tagesordnung und zwar unter Bedingungen, unter denen die Freiheiten und Institutionen erst geschaffen - und nicht verteidigt werden mussten.

Davon formell völlig verschieden stellt sich die Situation in der Bundesrepublik dar: Hier bedeutet die Erosion und Unterminierung des Rechtsstaates, der Weg in die Sicherheitsgesellschaft, dass gewachsene Identifikationen der Bürger mit ,allem was recht ist", eingespielte rechtsstaatliche Praxis, allmählich ausgehöhlt oder außer Kraft gesetzt werden. Der Angriff auf die Verfassungsordnung erfolgt auch in dem vorgezeichneten Szenario nicht durch eine Abschaffung des Grundgesetzes per Staatsstreich oder via Ermächtigungsgesetz, sondern graduell, zunächst offenbar in Form von ,rechtsstaatsverzehrenden Kontrollformen der Sicherheitsgesellschaft". Da weite Bereiche des öffentlichen und privaten Lebens von der Schwächung oder Aushebelung des Rechtsstaats zunächst nicht direkt betroffen sind, entzieht sich dieser Prozess weithin der Aufmerksamkeit der Öffentlichkeit. Es ist sogar damit zu rechnen, dass sich die Gesellschaft damit einrichtet. Das macht die Gefahr für den Rechtsstaat nicht geringer. Wenn wir also von einem Transformationsprozess ausgehen, dann bieten sich auf dem „Weg in die Sicherheitsgesellschaft" noch andere Mittel der politischen Auseinandersetzung an, als beim Kampf um Freiheitsrechte in einer etablierten Diktatur.

Wann die Schwelle zum Widerstand nach Art. 20 Abs. 4 des Grundgesetz erreicht ist, die den Einzelnen ermächtigt, zur Verteidigung der freiheitlichen Ordnung Widerstand $\mathrm{zu}$ leisten, ist in gewissem Sinne eine theoretische Frage, weil auch der Widerstand selbst, also politisches Handeln jenseits der noch existierenden verfassungsmäßigen politischen und rechtlichen Mittel, sich nicht übergangslos etabliert. Auch der Widerstand wird sich daran orientieren, welche legalen Ressourcen und Möglichkeiten noch auszuschöpfen sind und von passivem Widerstand über zivilen Ungehorsam zu aktivem Widerstand reichen - wobei insbesondere vor dem Schritt zur Anwendung von Gewalt gilt, dass ,andere Abhilfe nicht möglich ist“".

Welche Erfahrungen des politischen, gewaltfreien Widerstands in der DDR wären nun hier zu bedenken? Diese Frage hier zu stellen ist durchaus angemessen, wenn wir der etwas vereinfachten Sicht folgen, dass die Friedliche Revolution von 1989 in der DDR vor allem ein Sieg über die Staatssicherheit war. Bis heute gilt dieser Aspekt als das revolutionäre Vermächtnis von 1989, die Überwindung eines Systems, das sich auf eine zuvor nicht gekannte Überwachung und auf die damit ausgelöste direkte und indirekte Einschüchterung seiner Bürger stützte. So versteht sich, dass der Sieg über die Staatssicherheit bis heute auch symbolisch durch die Öffnung der Geheimdienstakten demonstriert wird, also in einer für jeden zugänglichen Praxis der Demontage des auf Geheimhaltung beruhenden Herrschaftswissens des Staates. Die gewonnene Freiheit symbolisiert sich also im Akt der Destruktion der Staatssicherheit. Insoweit passt das Beispiel. 
Diesem letztlich gelungenen „Weg aus der Sicherheitsgesellschaft“ ging sozusagen ein spiegelbildlich umgekehrter Prozess zum „Weg in die Sicherheitsgesellschaft" voraus: ausgehend von zunächst illegalen Aktionen des zivilen Widerstands und Ungehorsams einzelner Personen und Gruppen, über die Organisation von oppositionellen Netzwerken bis zur immer breiteren „Bürgerbewegung“ und Massenprotesten, durch die das System seine Macht über die Gesellschaft verlor.

Was in der DDR schon zu Opposition oder Widerstand zu rechnen war, erzeugt bis heute Definitionsprobleme. Eine Enquête-Kommission des Bundestages versuchte es in den 90er Jahren mit der Formel „Möglichkeiten und Formen abweichenden und widerständigen Verhaltens und oppositionellen Handelns“. Dies schloss alle Formen aktiver und passiver politischer Resistenz ein, praktisch konnte sich am Ende jeder DDRBürger darin irgendwie wiedererkennen. Eine engere Definition - das aktive, organisierte und über einen längeren Zeitraum wirkende Handeln im ,politischen Widerstand“ - würde auf wenige Oppositionsgruppen in der DDR zutreffen, wobei viele bewusst vermieden, den Zweck ihrer Gruppenbildung als ,politischen Widerstand“ zu deklarieren.

Diese Zurückhaltung oder Vorsicht half allerdings wenig, denn unabhängig vom Selbstverständnis der Akteure entschied die Definitionsmacht von Partei und Staat ohnehin darüber, was Opposition war. Die entsprechenden willkürlichen Vorlagen lieferten das 1979 erneuerte Strafgesetzbuch der DDR oder die einschlägigen Bestimmungen des MfS zur Bekämpfung „Politischer Untergrundtätigkeit“. Deren ebenso umfassende wie unscharfe Definitionen politischer Opposition waren nicht zuletzt darauf gerichtet, jegliches Legalitätsbewusstsein der Akteure zu untergraben und staatliche Repression nach Bedarf zu ermöglichen. ${ }^{9}$

Wie konnten sich unter diesen Umständen politische Selbstorganisation und - in Kenntnis des staatlichen Feindbildes - ein Selbstverständnis von Legitimität entwickeln, das für eine tragfähige und nachhaltige politische Opposition unverzichtbar ist?

9 Besonders der $\S 106$ Staatsfeindliche Hetze gehört hier her: Hier hieß es u.a.: „,Wer mit dem Ziel, die sozialistische Staats- und Gesellschaftsordnung... zu schädigen oder gegen sie aufzuwiegeln 1. Schriften, Gegenstände und Symbole, die die staatlichen, politischen, ökonomischen oder anderen gesellschaftlichen Verhältnisse... diskriminieren, einführt, herstellt, verbreitet oder anbringt... wird mit einer Freiheitsstrafe von einem Jahr bis zu fünf Jahren bestraft. "Entsprechend sahen auch die einschlägigen Bestimmungen des MfS zur Bekämpfung „Politischer Untergrundtätigkeit“ aus. Sie definieren diese im „Wörterbuch der politisch-operativen Arbeit"von 1981 als die ,, durch den konzentrierten Einsatz der politisch-ideologischen Diversion inspirierte und von feindlichen Zentren, Organisationen und Kräften organisierte Suche, Sammlung und Zusammenführung von feindlich-negativen Kräften zur Schaffung einer personellen Basis in der DDR, die in Durchsetzung feindlicher Plattformen unter Anwendung konspirativer Mittel und Methoden langfristig orientierend gegen die DDR mit dem Ziel kämpfen,... sozialismusfeindliche Positionen zu schaffen, Bürger... aufzuwiegeln, feindliche Handlungen zu aktivieren... “. Das MfS traf seit 1979 die Unterscheidung zwischen „feindlich-negativem Personenzusammenschluss" und „feindlich-negativer Gruppe“. Schon der lose Zusammenschluss war ebenfalls seit1979 durch § 218 ,Zusammenschluss zur Verfolgung gesetzwidriger Ziele“ unter Strafe gestellt worden. „Staatsfeindliche Gruppen“ waren schon dadurch definiert, dass sie z.B. auf ,das Erarbeiten, Übernehmen und Verbreiten antisozialistischer Konzeptionen, Plattformen, Schriften, Gegenstände und Symbole“ zielten. 
Der englische Historiker Ian Kershaw erklärt es so: „Die Art der Herrschaft bestimmt die Art des Widerstandes; je umfassender der Herrschaftsanspruch, desto mehr... Widerstand ist die Folge, denn das Regime selbst verwandelt Verhalten und Aktionen in Widerstand, die unter normalen Bedingungen... häufig überhaupt keine politische Bedeutung beanspruchen könnten." Das heißt, es galt zunächst die unterschiedlichsten Aktionsformen zu probieren, um direkte staatliche Unterdrückung zu unterlaufen und gleichzeitig das individuelle Ohnmachtsbewusstsein aufzuheben. Diese reichten von der legalen schriftlichen Beschwerde („Eingabe“) bis zum spontanen, unangemeldeten Straßenfest, vom Lesezirkel bis zum Fahrradkorso. Erst die folgenden Verbote und Unterdrückungspraktiken des Staates machten daraus Akte des zivilen Ungehorsams, des symbolischen Protestes oder Formen von Gegen-Öffentlichkeit, also Opposition.

Die dadurch erfolgte Politisierung von alltäglichen Lebensformen und Lebensfragen sowie die Inanspruchnahme der offiziell geltenden politischen Grundsätze - wie Frieden, Gerechtigkeit, Solidarität mit der Dritten Welt - stärkten einerseits das Legitimitätsbewusstsein der Gruppen, sie waren andererseits Schlüssel für die Formulierung gesellschaftlicher Alternativen. Im Umgang mit den Widersprüchen, mit Ungerechtigkeiten und Unrecht galt es Alternativen einzuüben. Sofern Widerspruch plausibel und unabweisbar war, Verweigerung auf konkrete Umstände bezogen und zugleich symbolisch, wurde Widerstand ein Akt des common sense, der moralischen Selbstverständlichkeit. Die Überschreitung der bestehenden Regeln, Sprachregelungen oder „No-GoAreas" unterhalb der Schwelle der direkten Konfrontation bedeuteten konkrete Freiheitsgewinne, ohne den Systembruch zu thematisieren. Der Weg war gewissermaßen auch hier das Ziel: Angstfreiheit durch Kompetenz, durch Agieren in Gruppen und Solidarität.

Allgemein kann daher sicher gelten: Bevor es möglich ist, gegen die Gefahren eines Sicherheitsstaates Widerstand zu mobilisieren, müssen innergesellschaftlich, bei den Bürgern selbst, Legitimitätsreserven aktiviert werden, die gegen die herrschende Perversion der Legalität in Anschlag gebracht werden können. Dazu braucht es Informiertheit, Publizität, Organisiertheit und Praktikabilität, aber auch die Konzentration auf einen bestimmten Widerspruch, ein Unrecht, das die Aktionen unabweisbar macht und breite Kreise solidarisiert. Der Durchbruch in der DDR war dann erreicht, als der Übergang von „Exit“" zu „Voice“ erfolgte, von Flucht zu Widerspruch, und zwar massenhaft. ${ }^{10}$ Es war der Wechsel vom Slogan der Ausreisewilligen „Wir wollen raus!“ zum Ruf ,Wir bleiben hier!“, der Umschlag zu Selbstbehauptung und Selbstbestimmung, zu „Wir sind das Volk!““

Was also wäre zu tun, um den „Weg in die Sicherheitsgesellschaft“ zu verhindern? Wenn es stimmt, dass die Erosion der Rechtssicherheit komplementär zur Erosion der sozioökonomischen Sicherheiten zunimmt, dass das daraus wachsende Klima der Unsicherheit zunehmend Sicherheitsbedürfnisse provoziert, mithin die Tendenz des Staates zum Ausbau von Prävention und repressiver Kontrolle befördert bzw. legitimiert, dann lässt sich das eine nicht ohne das andere lösen. Das heißt, die sozialen Probleme hinter dem wachsenden Sicherheitsproblem müssen in den Vordergrund gestellt werden,

10 Albert Otto Hirschman, Exit, Voice, and Loyalty. Responses to Decline in Firms, Organizations, and States, Cambridge MA 1970 (dt. 1974: Abwanderung und Widerspruch, Tübingen.). 
die Organisationen und Interessenvertreter der betroffenen sozialen Schichten müssen den Zusammenhang thematisieren. Dabei geht es darum, den Mechanismus der Abspaltung zu durchbrechen, der die Repression begünstigt: Weil die betroffenen Schichten oder Personenkreise an den „Rändern der Gesellschaft“ verortet werden, tendiert die sogenannte Mitte zu normativer Abspaltung. Die „Ränder“ werden zur Zielprojektion der Verdächtigung, Strafandrohung und Ausgrenzung, was die Bereitschaft zur Repression wiederum verstärkt.

Was geschieht nun? Bislang ist es immerhin ein zentrales Verfassungsorgan selbst, das die Einschränkung wichtiger Grundfreiheiten immer wieder zurückgewiesen hat. Den Tendenzen der Sicherheitsgesellschaft zu wehren, wäre auch die Sache für ein weites Feld zivilgesellschaftlicher Akteure. Deren Fähigkeit, sich auf diese Herausforderung einzustellen, könnte mit der neuen Erfahrung wachsen. Sind auch die Mittel und Möglichkeiten der politischen und publizistischen Aufklärung und Diskussion ausgeschöpft, dann bekommen zum Beispiel Aktionen des zivilen Ungehorsams, gewaltfreie symbolische Aktionen zur Durchbrechung der Feinbilder, der Bedrohungsängste und der Gefahrenprojektionen eine Möglichkeit, Bewusstsein für die Freiheitsgefährdung zu entwickeln.

Damit wäre man auf dem „Weg in die Sicherheitsgesellschaft“ bei einigen der genannten Erfahrungen und Praktiken angekommen, die den Widerstand in der DDR auf dem „Weg aus der Sicherheitsgesellschaft“ formte. Es wäre eine Art Wegkreuzung, von der aus ein Weg hinausführt, wenn es gelingt, öffentliches Bewusstsein und Teile der Machteliten umzustimmen.

So gesehen ist das Recht auf Widerstand nach Art. 20 Abs. 4 des Grundgesetzes ein gewisses Fundament, ein Stein des Anstoßes für ein Bewusstsein, das solchen Widerstand begründet und trägt, ein Freiheitsdenkmal letzten Endes.

\section{Widerstandsformen in der BRD (Karl-Heinz Dellwo $)^{11}$}

Ich war vor Jahren auf einer Veranstaltung im größten Centro Soziale in Rom, dem ehemaligen Schlachthof, ein von Linken und Migranten besetzter Ort. Dort diskutierten Richter aus dem ersten Moro-Verfahren mit Mitgliedern der Roten Brigaden über die Frage, welche inländischen und ausländischen staatlichen Kräfte während der MoroEntführung ein Interesse an einer militärischen Lösung hatten.

Ich habe mich gefragt, was ich hier beitragen kann, denn dieser Kreis ist für mich auch sehr ungewöhnlich und ich sehe meine Lage hier auch als prekär. Über Rechtskonzeptionen zu sprechen fühle ich mich nicht berufen. Ich möchte deshalb auch nur ein paar Überlegungen äußern zur RAF, zur Frage des Widerstandsrechts und zur politischen Bestimmung des Sicherheitsstaates, um hier einen Terminus von Herrn Albrecht aufzugreifen.

Auf die Kategorien des Widerstandsrechts im Grundgesetz hat sich die RAF nicht bezogen. Es gab in der RAF eine Auseinandersetzung zu dieser Frage, als Ulrike Mein-

11 Vgl. hierzu auch Bibliothek des Widerstands, 2. Juni 1967 (Band 1), Angela Davis (Band 2), Schrei im Dezember. Griechenland 2008 - Ein erschossener Demonstrant (Band 3) - alle drei Bänder herausgegeben von Willi Baer, Carmen Bitsch und Karl-Heinz Dellwo, Laika-Verlag, Hamburg 2010. 
hof in einem Entwurf die Begrifflichkeit von ,grundgesetzunwert erklärten Minderheiten" zur Diskussion stellte. Dieser Bezug wurde in der Diskussion verworfen. Die RAF hat es abgelehnt, sich auf das Widerstandsrecht im Grundgesetz zu beziehen, da sich dieses auf den Erhalt der Verfassung bezieht. Die Verfassung schützt die bürgerliche Norm, die den Kapitalismus zur Grundlage hat. Den aber hat die RAF grundsätzlich bekämpft und sich selbst gegenüber dem bestehenden politischen, staatlichen und wirtschaftlichen System als Fundamentalopposition definiert. Revolutionäre wollen über das Bestehende hinaus. Jenseits dieses politischen Transformationsbedürfnisses umfasst die Verfassung der BRD eine Wertnorm, die gegen jeden Rückschritt zu verteidigen ist.

Die RAF hatte einen Weltbezug, eine internationalistische Vorstellung, wie die meisten Linken damals. Ihr Bezugspunkt war auf der einen Seite der westliche Imperialismus, der damals nicht nur in Vietnam einen unrechten Krieg führte. Auf der anderen Seite bezog sie sich auf die kämpfenden Völker und das entstandene Bedürfnis in den Metropolen nach radikaler Umwälzung, nach Abschaffung von Ausbeutung und Strukturen, die die Menschen entfremden. Das war eine neue, linke Weltbewegung, ein reales Subjekt, ein geschichtsmächtiges Subjekt.

Akteure im Vietnamkrieg wie der damalige US-Verteidigungsminister McNamara haben ihm später jede Legitimation entzogen. Heute gilt der Vietnamkrieg allgemein als Verbrechen. Ich möchte deswegen hier die Frage aufwerfen, ob diese nachträgliche Delegitimierung nicht notwendigerweise auch Auswirkungen für die politische Bewertung der RAF hat? Gegen diesen Kriegs-Imperialismus zu stehen, der Millionen Tote $\mathrm{zu}$ verantworten hat - das war keine Frage irgendeines juristischen Widerstandsrechts, sondern eine Frage der Zugehörigkeit: Auf welcher Seite der Welt möchte man stehen? In der Bundesrepublik Deutschland mit seinen integrierten Nazi-Eliten war die Beantwortung dieser Frage damals aus meiner Sicht fast zwangsläufig. Hier konnte es nur die Trennung und den Bruch geben. Zugehörig fand man sich zu all denen auf der Welt, die vor Ort ihre bitteren ökonomischen und gesellschaftlichen Strukturen durchbrechen wollten und dabei von dem bestimmt waren, was wir selber wollten: praktisch und eindeutig solidarisch zu sein mit allen um Befreiung von egal welchen Zwängen kämpfenden Menschen in der Welt. Dort lag ein sozialer Sinn.

Der bewaffnete Kampf der RAF ist in den offiziellen Positionen des Staates immer in eine Richtung interpretiert worden: als Ausdruck von Verrückten, von Kranken, von Irrationalen, von bösen Menschen, von Kriminellen sowieso oder was auch immer. Nur eines war er dort nie: Ausdruck einer anderen politischen Vorstellung über das, was die Grundlage der Gesellschaft sein soll.

Abgesehen davon, dass wir - vom Standpunkt der bürgerlichen Norm - notwendigerweise auch deren Gesetze gebrochen haben, ihnen gegenüber also nie legal sein konnten: Wir waren nicht verrückt, wir waren nicht irrational, schon gar nicht waren wir unpolitisch. Wir waren wie viele andere in der Welt davon überzeugt, dass die historische Möglichkeit für ein anderes System als der Kapitalismus herangereift sei.

Das war keine Meinung. Das hatte eine Gewissheit im Hintergrund, die sich aus der Mächtigkeit des damals realen gegengesellschaftlichen Bewusstseins ergab. Die Linke hatte die Erstarrung der postfaschistischen Tabugesellschaft durchbrochen und neue emanzipatorische Tatbestände geschaffen. Man konnte mit diesem System damals nicht leben. 
In diesem Zusammenhang möchte ich aus einer Auseinandersetzung zwischen Herbert Marcuse und Theodor Adorno zitieren. Adorno, der Philosoph der Aufklärung, rief als Reaktion auf eine Institutsbesetzung durch seine Studenten 1968 die Polizei und ließ das Institut räumen. Eine Aufklärung, die einen Knüppel braucht, um sich zu behaupten, delegitimiert sich selbst. Marcuse widersprach Adorno in diesem Kontext, nicht in dessen politischer Analyse der gesellschaftlichen Verhältnisse, sondern mit einem einfachen, aber eindeutigen Hinweis: Er sehe bei seinen Studenten, schreibt er, eine „physisch-psychische Unmöglichkeit" sich in die vorhandenen gesellschaftlichen Verhältnisse zu integrieren. Und deswegen war es zwingend für ihn, sich auf die Seite der Jugend zu stellen und die gesellschaftlichen Verhältnisse zu verwerfen.

Wenn das den gesellschaftlichen Zustand zwischen Minderheiten und Mehrheiten charakterisiert - wo liegt dann die Legitimation? Gewiss nicht selbstverständlich bei denen, die die gesellschaftlichen Machtpositionen inne hatten. Mitte der sechziger Jahre sind die latent vorhandenen Brüche in der Gesellschaft politische geworden und radikal geworden.

In der Erfahrung von Verstrickung und Verlogenheit hilft oft nur Radikalität, um die Schleier wegzureißen und die Tatbestände klar zu sehen.

Die Entwicklung von Protest und Widerspruch zur Militanz und zum bewaffneten Kampf in der BRD ist schnell verlaufen. Warum war das so? Diese „schnelle Entwicklung“" spricht davon, dass es eine gesellschaftliche Prädisposition dafür gab. Diese Entwicklung muss man zwar auch in einem weltweiten Kontext verorten. Denn überall in der Welt gab es bewaffnete antiimperialistische Kämpfe. Aber sie waren in den ehemaligen faschistischen Staaten - Japan, Italien, BRD - von besonderer Härte. Das Bedürfnis nach Bruch mit den alten Eliten und dem restaurierten Kapitalismus war hier besonders groß.

Mein Genosse Lutz Taufer hat das mal als Bedürfnis für einen nachholenden antifaschistischen Kampf bewertet. Das ist sicher ein Aspekt einer vielschichtigen Wahrheit, in der wir gewiss auch nicht richtig lagen oder „Recht hatten“. Recht zu haben ist allerdings auch keine interessante Position.

Ohne das Vorhandensein dieser gesellschaftlichen Disposition zur gewaltsamen Entladung wäre keine der bewaffneten Gruppen auch nur für kurze Zeit so wirkungsmächtig gewesen, wie sie es über viele Jahre waren.

Wenn die Brüche in der Gesellschaft bewusst und also vollzogen sind, gibt es nichts mehr, was die Seiten miteinander verbindet. Die eigene Sache wird vollstreckt. Die andere Seite existiert nicht mehr jenseits eines nüchternen Feindbezuges. Das können Sie in Stockholm genauso sehen wie im toten Trakt von Köln Ossendorf, dem Durchziehen der Isolationshaft bis zum Tod von Holger Meins oder Siegfried Debus oder in der Unterdrückung des Politischen bei den gefangenen RAF-Mitgliedern in den Prozessen, exemplarisch in Stuttgart-Stammheim. Alle Angeklagten dort waren am Ende tot. Das ist ein Ende, das alle Seiten kennzeichnet.

Wenn ich über ein Widerstandsrecht nachdenke, ist das für mich an bestimmte politische und soziale Inhalte geknüpft. Diese Inhalte haben etwas mit der sozialen Emanzipation des Menschen zu tun. Ich finde, es gibt ein eindeutiges Widerstandsrecht gegen die Globalisierung des Kapitalismus, gegen neoliberale Konzepte, die von Darwin analysierte bewusstlose Naturverhältnisse wie „Auslese“ und „,der Stärkere setzt sich durch“ zur Grundlage der Gesellschaft machen wollen. Wer auf dieser Ebene des ökonomischen 
Darwinismus agiert, ist auf einer Ebene der nationalsozialistischen Ideologie angekommen. Nach dem 2. Weltkrieg gab es eine personale Kontinuität der nationalsozialistischen Eliten im Bestimmungsraum des neuen Systems. Heute, obwohl die meisten Altnazis verstorben sind, dringen Teile der nationalsozialistischen Ideologie ins Zentrum des Systems. Nicht nur im Personellen, sondern auch im Inhaltlichen hat sich vieles aus dem Nationalsozialismus in die BRD gerettet. Gerade auch am Inhaltlichen des Nationalsozialismus hat es keine radikale Kritik gegeben.

Es gibt kein Recht, dass den Einen ein höherwertiges Leben zusteht als den Anderen. Das lehne ich kategorisch ab. Das ist keine ideelle oder moralische Ablehnung. Sie kommt aus dem Wissen, dass in einer hochdifferenzierten, arbeitsteiligen Gesellschaft niemand mehr ohne den anderen irgendetwas auf dem entwickelten Niveau der Produktivkräfte bewirken kann. Der zentrale Maßstab, der heute zählen müsste, ist die Zeit, die man für die Gesellschaft, für das Gemeinsame erbringt. Dieser Maßstab muss sicher noch ausdifferenziert werden, aber dieses Ausdifferenzieren rechtfertigt nicht diesen obszönen Unterschied zwischen Armut und Reichtum, wie er heute die ganze Welt kennzeichnet.

Die Grenzen der Demokratie sind nicht einfach nur räumlich - wie im Begriff der „Festung Europa“ ausgedrückt. Sie sind inhaltlich und dieser Inhalt enthält den Ausschluss von geschichtlich möglicher Gleichheit für die Mehrheit der Menschen. Dieser Ausschluss hat den Sicherheitsstaat zur Folge und zur Bedingung. Aber er hat auch den Hass zur Folge von einer Mehrheit der Menschheit, die wissen, dass ihr legitimes Interesse nach konkreter Gleichheit auch im ökonomischen darin nicht mehr vorkommt. Ob die europäischen Bürger ihr System wollen, ist unbedeutend. Die Mehrheit der Welt ist davon ausgeschlossen oder kann sich die Privilegien Europas nur gegen die alten Mächte erkämpfen.

Der Systembezug, der Stand hält, muss einen Weltbezug haben. Europa - wie ebenso die USA - ist heute zur restlichen Welt wie der Adel gegenüber dem Bürgertum und wird genauso wenig bestehen. Die Hegemonie der alten kapitalistischen Zentren über die Welt ist verloren. Die wird über Kriegsstrategien wie die in Afghanistan nicht zurückerobert. Sie werden, wie früher in der Entkolonialisierungsphase nur eine weitere Brutalisierung in der Welt bringen, um dann doch am Ende ihre auf ökonomische und soziale Ungleichheit aufgebauten Positionen räumen zu müssen. Der Sicherheitsstaat ist hier nur eine Sicherheitsillusion. Wie immer begreifen die, die ihre Privilegien aus einer nur für sie nützlichen Ordnung ziehen, nicht, dass ihr Legitimationswolkenheim von anderen bereits weggeblasen ist. Die Karten über die Zukunft werden woanders gemischt. Die Mehrheit der Welt hat gegenüber der Minderheit der Reichen ein Widerstandsrecht.

\section{Zur Erforderlichkeit und Angemessenheit legitimer Widerstandsformen gegen die Sicherheitsgesellschaft (Michael Voß)}

Es stellen sich zusammenfassend zwei Fragen, nämlich einmal nach der Erforderlichkeit politischen Widerstandes gegen Sicherheitsgesetze und zum anderen nach der Angemessenheit und Legitimität jener Widerstandsformen in der Sicherheitsgesellschaft. 


\section{Ist „politischer Widerstand" gegen die Sicherheitsgesellschaft neben der Anrufung von Gerichten gegen einzelne Sicherheitsgesetze überhaupt erforderlich?}

1. Politischer Widerstand ist erforderlich, um die von Sicherheitsgesetzen Betroffenen zu sensibilisieren, auch als Gegengewicht gegen die permanente Anrufung des starken Staates in den Massenmedien.

2. Politischer Widerstand ist ferner erforderlich, um die gesetzgeberischen Produzenten und wissenschaftlichen Verteidiger von rechtsstaatsbedrohlichen Sicherheitsgesetzen zu delegitimieren, ihre Selbstgewissheit in Frage zu stellen.

3. Politischer Widerstand ist auch erforderlich, um den wenigen Rechtsstaatsverteidigern, die Gerichte anrufen, den Rücken zu stärken.

Die präventive Sicherheitsordnung in der „Sicherheitsgesellschaft“ bricht radikal mit der politischen Philosophie des liberalen Rechtsstaats. Statt Machtbegrenzung durch exakte Festlegung der Eingriffsvoraussetzungen tritt nun das Gegenteil ein: Die Straftatbestände werden unablässig vermehrt, die Strafdrohungen verschärft und die Strafzumessungsvoraussetzungen vereinfacht. Es entsteht ein „Strafrecht der Daseinsfürsorge“, wie es der Frankfurter Strafrechtler Wolfgang Naucke beklagt. Mit den Mitteln des Strafrechts werden soziale Probleme allerdings nur auf symbolische Weise „gelöst“. Staatliche Eingriffe sind über das Definitionsmonopol der Sicherheitsorgane gut kontrollierbar und - anders als wohlfahrtsstaatliche Maßnahmen der realen Problembehebung - außerordentlich kostengünstig. Und die Intervention verbleibt, anders als im Bereich der Ökonomie, wo man auf globale Verflechtungen und nicht-beeinflussbare Faktoren angewiesen ist, im nationalstaatlichen Zugriff. ${ }^{12}$

Doch wie reagiert der Bürger, der durch unsichere Arbeitsverhältnisse, nicht mehr finanzierbare Sozialsysteme, einen zumindest in den Medien allgegenwärtigen Terrorismus verunsichert ist: Diese verunsicherten Menschen suchen nicht Schutz vor dem Staat, sondern Schutz durch den Staat. Der ängstliche Bürger fürchtet sich nicht vor der Exekutive. Die Beseitigung rechtsstaatlicher Grundprinzipien erfolgt vielmehr häufig genug mit seiner Zustimmung. Die „Sicherheitsgesellschaft“ wird vielfach nicht als Bedrohung, sondern als Beruhigung der angespannten Bürgernerven empfunden. Und der Anspruch des ,ängstlichen Bürgers“ ist mittlerweile weit über die Forderung nach Sicherheit vor Gewalt hinausgewachsen, nämlich auf eine allumfassende präventive Sicherheit vor allen Lebensrisiken gerichtet.

Dieser Sachverhalt macht Widerstand gegenüber einer solchen Rechtsentwicklung zu einem gleichsam „demokratischen“ Problem. Der Verteidiger des liberalen Rechtsstaats hat nämlich nicht nur einen erheblichen Teil der Sicherheitsorgane und des Strafverfolgungssystems gegen sich, die jede Anrufung des Bundesverfassungsgerichts als Sabotageakt gegen ihren Kampf um Sicherheit sehen. Vielmehr hat der Verteidiger des Rechtsstaats vermutlich auch einen nicht geringen Teil der Bürger gegen sich. Unter diesem Aspekt erscheint das eher ,elitäre“ Bundesverfassungsgericht in der Tat als deutlich aussichtsreicheres Abwehrmittel als etwa das basisdemokratische Institut des Volksbegehrens. Bei Letzterem könnte der Rechtsstaat auf der Strecke bleiben.

12 Siehe Karl-Ludwig Kunz, Strafrechtsmodelle und Gesellschaftsstruktur, in: Kriminologisches Journal, 1/2010, S. 16 ff.; Trutz von Trotha, Die präventive Sicherheitsordnung, in: Kriminologisches Journal, 1/2010, S. $35 \mathrm{ff}$. 
Das ist eine Schlussfolgerung für Widerstandshandlungen, die auch aus den historischen Erfahrungen gespeist wird: Widerstand kommt nicht selten als höchst elitäre oder avantgardistische Initiative zustande (siehe den Widerstand im Nationalsozialismus), tritt - zumindest zu Beginn - zunächst gegen die Mehrheitsmeinung an und hat insoweit häufig gegen konkurrierende Deutungen wie „Verrat“, „kriminelle Handlung“ oder „nützlicher Idiot des Feindes“ zu kämpfen - das Elser-Attentat oder die Freiheitsbewegung in der DDR liefern hier reichhaltiges Anschauungsmaterial.

Aus diesem Sachverhalt erwächst die Notwendigkeit, dass politischer Widerstand auf eine Art von Fundamentalkritik angewiesen ist, zu einer bewussten Dramatisierung der Lage greifen muss, um wachzurütteln und die klammheimliche Zustimmung des Bürgers zum „starken Staat“ zu durchbrechen. Insofern braucht es - um ein anderes Politikfeld zu nennen - Klimaforscher, die bereits heute die Klimakatastrophe in ihren letzten furchtbaren Auswirkungen beschreiben oder Juristen, die bereits heute den gänzlichen Verlust des Rechtsstaates zu Ende denken. Nur so lässt sich politischer Widerstand mobilisieren. Also: wir brauchen politische Gegenstrategien, wir dürfen nicht nur auf die Gerichte vertrauen.

\section{Was sind angemessene und legitime Widerstandsformen gegen die Sicherheitsgesellschaft?}

1. Selbstverständlich muss es auch bei der Ausschöpfung der Rechtsmittel bleiben, bis hin zur übernationalen Gerichtsbarkeit (Beispiel: Europäischer Gerichtshof für Menschenrechte zur Sicherungsverwahrung). ${ }^{13}$

2. Auch die empfohlene Stärkung der Dritten Gewalt als Gegengewicht zu dem Überschwang der Exekutive, also die Stärkung der gerichtlichen Kontrollfunktionen, gehört zu den angemessenen politischen Gegenmitteln. ${ }^{14}$

3. Darüber hinaus tut sich ein großes Feld von fantasievollen politischen Widerstandsstrategien auf, die vom Einsatz wissenschaftlicher und publizistischer Mittel an dem einen Ende bis hin zum Widerstandsrecht und seinen gewaltsamen Eventualkomponenten am anderen Ende reichen. All das zielt auf die Wiederherstellung des Rechtsstaats. Über dieses Spektrum hinaus führen Widerstandsüberlegungen, die die strukturellen gesellschaftlichen Gründe für das Anwachsen des Sicherheitsbedürfnisses und das Anwachsen der Sicherheitsgesetze selbst beseitigen wollen.

Gewalt ist kein Ersatz, kein alternatives Mittel für politische Kommunikation, sondern meint schlicht den Abbruch zivilisierter Umgangsformen, wodurch auch Politik als Problemlösungsansatz im Grunde ausgehebelt wird. Es ist insofern ein unpolitisches Instrumentarium. Dabei könnte man es bewenden lassen. Gleichwohl kann man nicht ignorieren, dass Gewalt allenthalben ausgeübt wird, beileibe nicht nur im Sinne der Straßenkriminalität - sozusagen im zwischenmenschlichen Bereich. Vielmehr sind die weltweit großen Gewalteruptionen

- dort zu finden, wo Staaten gegen andere Staaten zu Felde ziehen, um sich deren Land, deren Ressourcen zu bemächtigen oder um eine ihnen genehme Regierung zu installieren,

13 Vgl. EGMR StV 2010, S. $181 \mathrm{ff}$.

14 Siehe Peter-Alexis Albrecht, Der Weg in die Sicherheitsgesellschaft, 2010, S. 989 ff. 
- sie sind dort zu finden, wo staatliche Armeen ihre Bürger unterdrücken oder Unterdrückte sich gegen den Staat auflehnen,

- sie sind dort zu finden, wo Gläubige gegen Ungläubige oder Andersgläubige einen Kreuzzug führen,

- sie sind dort zu finden, wo Arme gewaltsam in die geschützten Gefilde der Reichen eindringen oder wo diese wiederum ihre Privilegien gewaltsam gegen Arme verteidigen usw.

All diese permanente Gewaltausübung, die uns täglich auf dem Bildschirm vor Augen geführt wird und an der mittlerweile auch unsere Gesellschaft beteiligt ist, hat natürlich hochgradig politische Konsequenzen. Sie wirkt zum einen geradezu als angsterzeugender Katalysator in Richtung der heute beklagten Sicherheitsgesellschaft. Und sie verweist in ihrer Allgegenwart auf die der Gewalt zugrunde liegende soziale Ungerechtigkeit. Nichts anderes hat Karl Heinz Dellwo angemerkt, wenn er Gleichheit und Gerechtigkeit zwischen den Menschen einfordert und dabei einen „Weltbezug“ herstellt. Das ist in der Analyse vollkommen richtig. Bis man allerdings die geeigneten Mittel gefunden hat, Gleichheit und Gerechtigkeit - noch dazu weltweit - herzustellen, wird man noch etwas überlegen und streiten müssen.

Eines ist jedenfalls klar: Gleichheit auf globaler Ebene kann angesichts der begrenzten Ressourcen der Erde und der Überbevölkerung nicht in Richtung des westlichen Wohlstandsniveaus für alle gedacht werden. Möglich ist nur ein „Downgrading“, eine Angleichung auf einem niedrigen Niveau des Lebensstandards, oder es muss eine ganz neue Definition von Lebensstandard geben, ohne den ressourcenverzehrenden Massenkonsum. Das bedeutet also, dass die westlichen Industrienationen Verzicht lernen müssen, lernen müssen, abzugeben. 